\title{
Diagnosis and Treatment of Inflammatory Joint Disease
}

\author{
Yeesuk Kim, MD, Hyun-Cheol Oh, MD*, Jang Won Park, MD, In-Sung Kim, MD${ }^{\ddagger}$, Jun-Young Kim, MD, \\ Ki-Choul Kim, MD", Dong-Sik Chae, MD", Woo-Lam Jo, MD**, Joo-Hyoun Song, MD ${ }^{++}$ \\ Department of Orthopaedic Surgery, Hanyang University Hospital, Seoul, Korea \\ Department of Orthopedic Surgery, National Health Insurance Service Ilsan Hospital, Goyang, Korea* \\ Department of Orthopaedic Surgery, Ewha Womans University Medical Center, Seoul, Korea ${ }^{+}$ \\ Department of Orthopedic Surgery, Hallym University Dongtan Sacred Heart Hospital, Hwaseong, Korea ${ }^{\ddagger}$ \\ Department of Orthopedic Surgery, Kyungpook National University Hospital, Daegu, Korea \\ Department of Orthopaedic Surgery, Dankook University Hospital, Cheonan, Korea" \\ Department of Orthopedic Surgery, International St. Mary's Hospital, \\ Catholic Kwandong University College of Medicine, Incheon, Korea" \\ Department of Orthopaedic Surgery, The Catholic University of Korea, Seoul St. Mary's Hospital, Seoul, Korea** \\ Department of Orthopaedic Surgery, The Catholic University of Korea, St. Vincent's Hospital, Suwon, Korea ${ }^{++}$
}

Arthritis damages the cartilage within joints, resulting in degenerative changes, including loss of function and joint instability. Ankylosing spondylitis (AS) is a chronic inflammatory condition affecting the spine and boneto-tendon attachment area within the sacroiliac joint leading to back pain and progressive spinal stiffness. In the final stages, AS causes hyperkyphosis-a condition closely tied to the human leukocyte antigen-B27 gene. Rheumatoid arthritis is a chronic, systemic autoimmune disease characterized by the simultaneous inflammation of the synovium of multiple joints, leading to joint damage (e.g., destruction, deformation and disability). In the past, nonsteroidal anti-inflammatory drugs or conventional disease-modifying antirheumatic drug (DMARDs) have been used for the treatment of these autoimmune diseases, but biologic DMARDs have recently been introduced with excellent results. Gout is a chronic inflammatory disease that causes an alteration of joints resulting in severe pain. Specifically, gout is associated with an accumulation of uric acid within the body resulting from dysregulated purine metabolism, causing recurrent paroxysmal inflammation in the joints. Allopurinol and febuxostat are the primary treatment options for individuals with gout. It is necessary to have an accurate understanding of the pathogenesis, pathological ecology and treatment of AS, rheumatoid arthritis, and gouty arthritis, which are the representative diseases that may cause inflammatory arthritis.

Key Words: Reactive arthritis, Ankylosing spondylitis, Rheumatoid arthritis, Gout

Submitted: August 18, 2017 1st revision: November 6, 2017

Final acceptance: November 7, 2017

Address reprint request to

Joo-Hyoun Song, MD

Department of Orthopaedic Surgery, The Catholic University of

Korea, St. Vincent's Hospital, 93 Jungbu-daero, Paldal-gu, Suwon

16247, Korea

TEL: +82-31-249-7114 FAX: +82-31-254-7186

E-mail: osong97dayahoo.co.kr
This is an Open Access article distributed under the terms of the Creative Commons Attribution Non-Commercial License (http://creativecommons. org/licenses/by-nc/4.0) which permits unrestricted non-commercial use, distribution, and reproduction in any medium, provided the original work is properly cited. 


\section{Hip \& Pelvis}

Hip Pelvis 29(4): 211-222, 2017

\section{INTRODUCTION}

Arthritis is a disease that may cause damage to the healthy cartilage of joints, leading to degenerative changes, loss of function and joint instability. Inflammatory arthritis is known to be caused by an increase of cytokines which leads to degradation of articular cartilage and a decrease of growth factors which induce chondrogenesis. Although a variety of diseases may cause inflammatory arthritis, this study reviews the most common associated disorders (i.e., ankylosing spondylitis [AS], rheumatoid arthritis [RA], and gouty arthritis).

\section{ANKYLOSING SPONDYLITIS}

\section{Diagnosis}

AS is associated with chronic inflammation of the spine and bone-to-tendon attachment area of the sacroiliac joint that causes back pain and progressive spinal stiffness. At the final stage of AS, bony fusion and severe hyperkyphosis may occur. AS is a type of spondyloarthritis that may cause musculoskeletal symptoms (e.g., spondylitis, oligoarthritis, enthesitis, dactylitis), and non-musculoskeletal symptoms (e.g., anterior uveitis, psoriasis, inflammatory bowel disease).
This condition is closely linked to the human leukocyte antigen (HLA)-B27 gene ${ }^{1,2}$. AS is subdivided into axial and peripheral types based on whether symptoms chiefly affect the spine or the peripheral joints, and it can be diagnosed when sacroiliitis is clearly present in axial type. All disorders classified as spondyloarthritis can progress to AS.

AS, with a median onset age of 23 years, usually begins between the ages of 10 to 20 years and almost always prior to the age of 40 years; only $5 \%$ of individuals experience initial manifestations after 40 years. The male:female ratio is reportedly 2 to $3: 1$. The prevalence of AS varies widely by race and region and it is directly related to and proportional to the prevalence of HLA-B27. Prevalence rates are reportedly higher in Europe and North America than in Asia and Africa. The prevalence of AS ranges between $0.2 \%$ to $1.2 \%$ (European whites), $6 \%$ to $9 \%$ (Central European populations) and less than $1 \%$ in Asia and Africa ${ }^{1,2}$. About $90 \%$ of patients with AS are positive for HLA-B27, and $1 \%$ to $6 \%$ of HLA-B27positive individuals are diagnosed with AS. These findings suggest that AS cannot be diagnosed based on HLA-B27 status alone.

Clinically, AS is characterized by inflammatory back pain (IBP), which is distinguished from mechanical back pain manifested in herniated intervertebral disc, muscle sprain and degenerative spinal disease. IBP is characterized by: i)

\section{ASAS classification criteria for axial SpA \\ (in patients with back pain $\geq 3$ months and age at onset $<45$ years)}

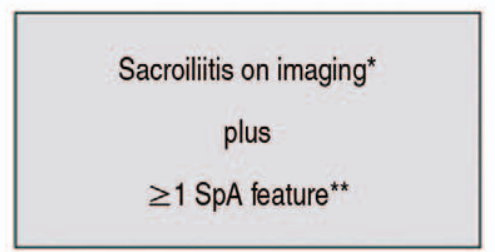

** SpA features:

- Inflammatory back pain

- Arthritis

- Enthesitis (heel)

- Uveitis

- Dactylitis

- Psoriasis

- Crohn's disease/ulcerative colitis

- Good response to NSAIDs

- Family history for SpA

- HLA-B27

- Elevated CRP

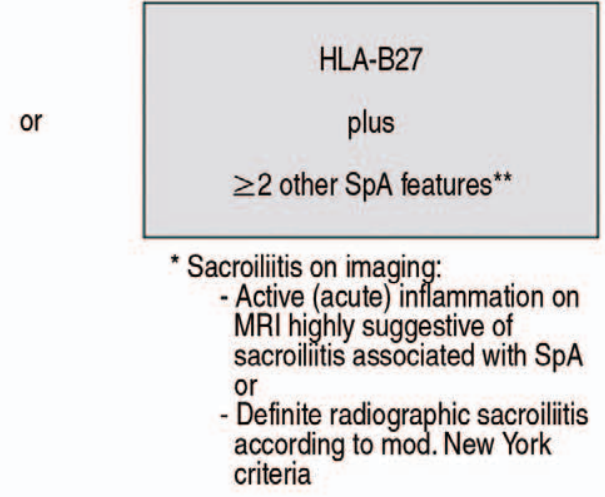

\section{Sensitivity $82.9 \%$, specificity $84.4 \% ; n=649$ patients with chronic back pain and age at onset $<45$ years. Imaging arm (sacroiliitis) alone has a sensitivity of $66.2 \%$ and a specificity of $97.3 \%$.}

${ }_{* *}$ Note: Elevated CRP is considered a SpA feature in the context of chronic back pain

Fig. 1. Assessment of Spondyloarthritis International Society (ASAS) classification criteria for axial spondyloarthritis (SpA). HLA: human leukocyte antigen, MRI: magnetic resonance imaging, NSAIDs: nonsteroidal anti-inflammatory drugs, CRP: Creactive protein.

Data from the article of Rudwaleit M et al. (Ann Rheum Dis 2009;68:777-83) ${ }^{8}$. 
onset before the age of 40 years after a symptom period of more than 3 months, ii) insidious onset, iii) improvement with exercise, iv) no improvement during rest periods, and v) nocturnal symptoms. Furthermore, morning stiffness for more than 30 minutes and bilateral hip pain may be associated alternately ${ }^{2,3)}$. Unlike mechanical back pain, IBP appears to resolve within 24 to 48 hours with the use of anti-inflammatory drugs. There can be limits to motion in the spine and chest as AS progresses. For diagnosis of AS, there are no specific laboratory findings, erythrocyte sedimentation rate (ESR)/ C-reactive protein (CRP) can be raised and tests for rheumatoid factor, anti-cyclic citrullinated peptides (CCP) and antinuclear antibodies are negative. Hip joint lesions are associated in more than $25 \%$ to $35 \%$ of patients with AS, and severe dysfunction and poor prognosis are typically seen. In particular, more severe hip lesions are manifested radiographically in cases of early onset and severe radiographic deformity in the spine and sacroiliac joint ${ }^{4-6}$.

The use of magnetic resonance imaging (MRI) and tumor necrosis factor (TNF)- $\alpha$ antagonists has enabled recent advances in the diagnosis and treatment of AS. This advancement has increased possibilities for early diagnosis and treatment of sacroiliitis unseen by plain X-rays. In the diagnosis of AS, IBP, motion restriction in the spine and chest and associated symptoms are examined clinically and the presence of sacroiliitis is confirmed radiologically. The modified New York criteria ${ }^{7}$ have also been used for the diagnosis of AS; however, a disadvantage associated with this system is that the presence of sacroiliitis is hardly identified in the early stage in the evaluation of plain X-rays. Several previous studies suggest that the duration from symptom onset to diagnosis of AS takes about 5 to 11 years ${ }^{2}$.

By modifying former diagnostic criteria and applying MRI's advantages, new criteria have been established. In 2009, the recent diagnostic criteria for axial spondyloarthritis had been proposed by the Assessment of Spondyloarthritis International Society (ASAS) by including clinical characteristics, MRI findings of the sacroiliac joint and HLA-B27 (Fig. 1). According to ASAS guidelines, among patients less than 45 years of age and having chronic pain for more than 3 months, those meeting one of the following criteria are defined as having AS: 1) clear findings of sacroiliitis are seen on plain $\mathrm{X}$-ray and MRI and at least one other feature of spondyloarthritis is concurrently associated; and 2) HLA-B27 positive and at least two other spondyloarthritis-associated features are fulfilled $^{8}$. In conclusion, the main clinical signs useful in the diagnosis of AS are IBP, the presence of sacroiliitis on Xray or MRI and HLA-B27(+).

\section{Treatment}

The aims of treatment are to reduce pain and stiffness in the affected joint, restore correct posture and improve quality of life by providing physical and cognitive function ${ }^{9}$. The most appropriate management of AS is a combination of pharmacological and non-pharmacological treatments ${ }^{10}$. Non-pharmacological methods have gained much attention since exercise and physical therapies have provided a wide range of benefits including pain relief and improvement in joint flexibility ${ }^{11}$. The primary goals of exercise therapy are to reduce joint stiffness, strengthen muscles around the affected joint, prevent joint deformity and functional loss and alleviate pain $^{12)}$.

Exercise therapy includes range of motion (ROM), musclestrengthening and aerobic exercises. ROM exercises are divided into passive, active-assistive, active and stretching. Strength training can be further classified into isometric, isotonic and isokinetic. ROM exercises and stretching specific for AS (AS specific: dynamic mobility and stretch therapy) are helpful in maintaining joint flexibility and good posture. Appropriate sleep and proper walking posture, back and abdominal exercises assist in maintaining upright posture. Moreover, exercise therapy should be considered for patients with AS, since spine and chest stiffness and decreased respiratory system functions and motor ability due to chest wall alterations may occur $^{13,14}$. Advantages of exercise therapy include: i) providing aerobic workout, ii) physical training, which promotes spine flexibility, strength endurance and pulmonary function ${ }^{12,15}$, and iii) reducing the risk of cardiopulmonary complications ${ }^{16}$.

Regular physical activity can improve health, well-being and functional outcomes of patients, and leisure activities such as Tai Chi, Pilates and swimming can be helpful in enhancing functional recovery ${ }^{17-19}$. Moreover, a combined treatment of TNF- $\alpha$ antagonist therapy and AS-specific exercise program showed better functional outcome than TNF- $\alpha$ antagonist or exercise therapy alone ${ }^{20)}$. Spa and ultrasound therapy and aquatic exercise are shown to be effective in relieving pain and improving functional recovery ${ }^{21-24}$. The frequency, intensity and duration of physical therapy should be appropriately adjusted for each individual based on their ability and lifestyle patterns. In addition, motivating and educating individuals to perform exercise constantly on a regular basis are warranted ${ }^{12)}$.

New clinical practice guidelines for management of AS are the 2016 ASAS/European League Against Rheumatism (EULAR) ${ }^{25}$ and the 2015 American College of Rheumatology (ACR)/Spondylitis Association of America 
(SAA)/Spondyloarthritis Research and Treatment Network $(\text { SPARTAN })^{26)}$. In the ASAS/EULAR recommendations, secukinumab, the interleukin (IL)-17 inhibitor, is already included as a treatment option.

The nonsteroidal anti-inflammatory drugs (NSAIDs) have been shown to be effective in reducing pain and spine stiffness. For this reason, NSAIDs are recommended as first line agents. Although definite differences in several types of NSAIDs have not yet been clarified, they are effective in both patients with AS and non-radiographic axial spondyloarthritis. These agents have been shown to be effective within the first two weeks of use and remain so for up to 24 weeks. NSAIDs are more effective in patients at the early stages of illness. However, there are insufficient data on side effects of longterm use of NSAIDs in patients with AS and their risks are determined based on previous studies on patients with osteoarthritis or RA.

Among disease-modifying antirheumatic drugs (DMARDs), conventional DMARDs such as methotrexate (MXT), sulfasalazine and leflunomide usually have no effect in individuals experiencing axial AS; however, they may have partial effect in individuals with peripheral AS. Furthermore, the combined use of conventional DMARDs and biological agents has recently emerged, but there is insufficient evidence to guide empirical treatment recommendations. Systemic glucocorticoid (GC) therapy is not recommended because a daily dose of prednisolone should be relatively high (i.e., doses greater than $50 \mathrm{mg}$ ) in order to impact the disease to a measurable degree.

Presently, there are five TNF inhibitors approved in the European Union (EU) and the United States (infliximab, etanercept, adalimumab, golimumab and certolizumab); each of these are effective in improving symptoms in the joints. These TNF inhibitors suppress immune response to TNF and lower inflammatory response. The use of TNF inhibitors for 24 weeks in patients with AS has been shown to: i) reduce joint pain, ii) increase functional recovery, and iii) induce partial remission ${ }^{27}$. Adverse events associated with the use of TNF inhibitors include severe infections, tuberculosis, cancer and others. Therefore, monitoring for tuberculosis should be performed before and during anti-TNF treatment. Patients receiving anti-TNF therapy need to be followedup cautiously because the risk of lymphoma or other solid cancers may be increased. Anti-TNF therapies are administered through injections, either in a hospital (infusion) or selfinjection at home (subcutaneous). The clinical efficacy of TNF inhibitors may rarely be seen within the first two weeks of use, but symptoms mostly improve after several months.
Elevated CRP levels, short symptom periods and active inflammation on MRI seem to be favorable prognostic factors related to treatment with TNF inhibitors. Among patients with a good response to anti-TNF therapy, about $75 \%$ to $90 \%$ experienced a relapse of symptoms after discontinuance. Meanwhile, decreased doses of TNF inhibitors maintained symptom control in $52 \%$ to $86 \%$ of patients. However, current evidence of their clinical effects is still unclear.

Among other biological agents, IL-1 receptor antagonist or T cell modulator have shown insignificant effects compared to placebo, whereas agents targeting the IL-23/IL-17 axis have had somewhat favorable results. Secukinumab, a monoclonal antibody targeting IL-17 has been shown to be effective in the treatment of AS and was recently approved in the EU and the United States for this indication. Though this IL-17 inhibitor appears to have partial efficacy in patients refractory to TNF-inhibition, its efficacy is at this stage unclear. The ASAS/EULAR and ACR/SAA/SPARTAN guidelines have acknowledged and recommended that hip replacement surgery should be considered for AS patients associated with hip arthritis.

\section{RHEUMATOID ARTHRITIS}

\section{Diagnosis}

RA is a systemic autoimmune disease characterized by the simultaneous inflammation of the synovium of multiple joints. This condition causes chronic damage of joints including destruction, deformation and disability. Its prevalence ranges between $0.3 \%$ to $2.1 \%$ in the general population, and it affects approximately $0.8 \%$ and $1.4 \%$ of the world and Korean populations, respectively.

The RA classification criteria were first introduced by the ACR as described Arnett et al. ${ }^{28}$ in 1987. The seven ACR 1987 criteria are as follows: 1) morning stiffness, 2) arthritis of 3 or more joint areas, 3) arthritis of hand joints, 4) symmetric arthritis, 5) rheumatoid nodules, 6) serum rheumatoid factor, and 7) radiographic changes. RA is defined by the presence of 4 or more criteria for at least 6 weeks. The ACR 1987 criteria cannot be entirely relied on for diagnosing RA at earlier stages. Thereafter, the ACR 1987 revised criteria for the classification of RA were established in $2010^{29}$. According to the ACR 2010 classification, cases fulfilling the 1987 criteria with the presence of joint synovitis are defined as RA (Fig. 2). Using the new 2010 criteria, the number and site of involved joints are scored on a 0 to 5 scale. Large joints consist of 
Yeesuk Kim et al. Diagnosis and Treatment of Inflammatory Joint Disease

the hip, knee, shoulder and elbow, whereas small joints include the wrist and hand. The scores are based on serological tests according to elevated levels of rheumatoid factor or presence of anti-CCP antibody (range, 0-3), according to CRP and/ or ESR (range, 0-1) and duration of symptoms (<6 weeks,
$0 ; \geq 6$ weeks, 1). Based on the sum of point scores in the four domains, a total score of 6 or greater out of 10 signifies RA. Patients with a score of less than 6 out of 10 require frequent monitoring.

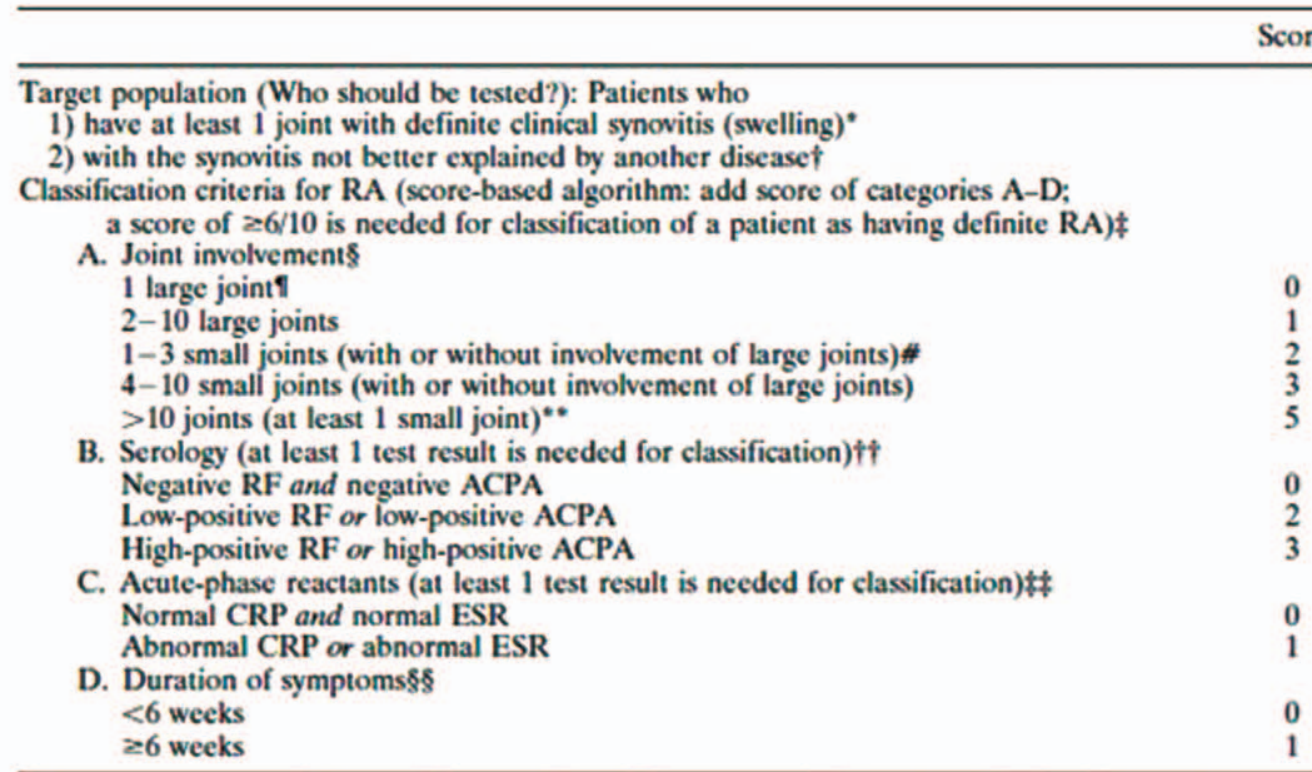

- The criteria are aimed at classification of newly presenting patients. In addition, patients with erosive disease typical of rheumatoid arthritis (RA) with a history compatible with prior fulfillment of the 2010 criteria should be classified as having RA. Patients with longstanding disease, including those whose disease is inactive (with or without treatment) who, based on retrospectively available data, have previously fulfilled the $\mathbf{2 0 1 0}$ criteria should be classified as having RA.

$\uparrow$ Differential diagnoses vary among patients with different presentations, but may include conditions such as systemic lupus erythematosus, psoriatic arthritis, and gout. If it is unclear about the relevant differential diagnoses to consider, an expert rheumatologist should be consulted.

$\$$ Although patients with a score of $<6 / 10$ are not classifiable as having RA, their status can be reassessed and the criteria might be fulfilled cumulatively over time.

$\$$ Joint involvement refers to any swollen or tender joint on examination, which may be confirmed by imaging evidence of synovitis. Distal interphalangeal joints, first carpometacarpal joints, and first metatarsophalangeal joints are excluded from assessment. Categories of joint distribution are classified according to the location and number of involved joints, with placement into the highest category possible based on the pattern of joint involvement.

I "Large joints" refers to shoulders, elbows, hips, knees, and ankles.

" "Small joints" refers to the metacarpophalangeal joints, proximal interphalangeal joints, second through fifth metatarsophalangeal joints, thumb interphalangeal joints, and wrists.

* In this category, at least 1 of the involved joints must be a small joint; the other joints can include any combination of large and additional small joints, as well as other joints not specifically listed elsewhere (e.g., temporomandibular, acromioclavicular, sternoclavicular, etc.).

it Negative refers to IU values that are less than or equal to the upper limit of normal (ULN) for the laboratory and assay; low-positive refers to IU values that are higher than the UL.N but $\leq 3$ times the UL.N for the laboratory and assay; high-positive refers to IU values that are $>3$ times the ULN for the laboratory and assay. Where rheumatoid factor (RF) information is only available as positive or negative, a positive result should be scored as low-positive for RF. ACPA = anti-citrullinated protein antibody. \# Normal/abnormal is determined by local laboratory standards. CRP = C-reactive protein; ESR = erythrocyte sedimentation rate.

$\$ \S$ Duration of symptoms refers to patient self-report of the duration of signs or symptoms of synovitis (e.g., pain, swelling, tenderness) of joints that are clinically involved at the time of assessment, regardless of treatment status.

Fig. 2. The 2010 American College of Rheumatology/European League Against Rheumatism classification criteria for rheumatoid arthritis.

Data from the article of Aletaha D et al. (Arthritis Rheum 2010;62:2569-81) ${ }^{291}$. 


\section{Treatment}

The goals of RA treatment are to relieve pain by controlling inflammation and to improve quality of life by preventing joint damage and maintaining physical function. Based on these principles, conservative treatment such as physical therapy, aspirin and NSAIDs was primarily used to manage RA in the past. As traditional stepped-up therapy, a pyramid approach was taken in patients with RA, beginning with DMARD agents considered relatively safe for those with no improvement of symptoms or joint damage and progressively more intense immunosuppressive agents. However, as these therapies often had no effect on joint damage, the trend has been changed to implement early treatment and early use of DMARDs. Thus, the ACR and EULAR recommend the use of DMARDs in the treatment of RA from diagnosis ${ }^{30,31}$. Customized treatment plans for each patient according to the progression and prognosis of RA are warranted. Early treatment is necessary because bone destruction progresses within the first two years of the disease. Therefore, the early diagnosis and early treatment of RA are crucial within the first 6 months after the onset of symptoms. In addition, a key treatment decision is to use intense drugs with the fewest side effects by screening RA patients with a rapid progress of the disease and poor prognosis.

Medications used in the treatment of RA include NSAIDs, GCs, DMARDs and others. Unlike in the conventional guidelines, the ACR recommends the use of GCs for patients with refractory RA in whom treatment with high-dose DMARDs has failed, and suggests that short-term use of GC treatment at a low dose of $10 \mathrm{mg}$ per day for less than 3 months is most effective. The EULAR recommends GCs be added at low doses of less than $7.5 \mathrm{mg}$, and GCs have become part of current combination regimens particularly at low doses in short-term. The most common agent of DMARDs is MTX, the conventional synthetic DMARD (csDMARD), and other agents include sulfasalazine, hydroxychloroquine, leflunomide and others. DMARDs are used alone or in combination with csDMARD, biologic DMARD (bDMARD) and targeted synthetic DMARD (tsDMARD). According to the EULAR guidelines, MTX is given once a week at a dose of 25 to $30 \mathrm{mg}$ for a maximum of 8 to 12 weeks depending on patient compliance. The maximum recommended dose to be given is $20 \mathrm{mg}$ in China and $16 \mathrm{mg}$ in Japan. bDMARDs are protein-based drugs that block inflammation and destruction of invaded tissues by restricting the function of proinflammatory cytokines or accelerating the function of anti-inflammatory cytokines. bDMARDs, including adalimumab, certolizumab, etanercept, golimumab, and infliximab, are TNF-inhibitors and most commonly used to treat csDMARD-refractory patients. Other bDMARDs, which target molecules other than TNF, include: i) abatacept, a costimulatory inhibitor that blocks the specific binding of CD80/CD86 to CD28 receptor, therefore, inhibiting $\mathrm{T}$ cell proliferation; ii) tocilizumab, an inhibitor of IL-6 receptor; iii) clazakizumab and sirukumab,

\begin{tabular}{|cl|}
\hline Instrument (reference) & \multicolumn{1}{c|}{ Thresholds of disease activity } \\
\hline $\begin{array}{c}\text { Patient Activity Scale (PAS) of PASII } \\
\text { (range 0-10) (149) }\end{array}$ & Remission: $0-0.25$ \\
& Low activity: $>0.25-3.7$ \\
& Moderate activity: $>3.7$ to $<8.0$ \\
Routine Assessment of Patient Index Data 3 & High activity: $\geq 8.0$ \\
(RAPID3) (range 0-10) (155) & Remission: $0-1.0$ \\
& Low activity: $>1.0-2.0$ \\
& Moderate activity: $>2.0$ to $<4.0$ \\
Clinical Disease Activity Indes (CDAI) & High activity: $>4.0-10$ \\
(range 0-76.0) (156) & Remission: $\leq 2.8$ \\
& Low activity: $>2.8-10.0$ \\
& Moderate activity: $>10.0$ to $<22.0$ \\
Disease Activity Score (DAS) 28 & High activity: $>22$ \\
erythrocyte sedimentation rate (ESR) & Remission: $<2.6$ \\
(range 0-9.4) (157) & Low activity: $\geq 2.6$ to $<3.2$ \\
Simplified Disease Activity Index (SDAl) & Moderate activity: $\geq 3.2$ to $\leq 5.1$ \\
(range 0-86.0) (158) & High activity: $>5.1$ \\
& Remission: $\leq 3.3$ \\
& Low activity: $>3.3$ to $\leq 11.0$ \\
& Moderate activity: $>11.0$ to $\leq 26$ \\
& High activity: $>26$ \\
\hline
\end{tabular}

Fig. 3. Definition and remission of rheumatoid arthritis disease presented by the American Society of rheumatoid diseases. Data from the article of Singh JA et al. (Arthritis Rheumatol 2016;68:1-26) ${ }^{30}$. 
Yeesuk Kim et al. Diagnosis and Treatment of Inflammatory Joint Disease

which target IL-6; iv) rituximab, a $\beta$-cell-depleting agent targeting CD-20; and others. Previous studies have reported that bDMARDs are up to $25 \%$ more effective when used concomitantly with MTX. The types of tsDMARDs are Janus kinase (JAK)-inhibitor tofacitinib and baricitinib. The EULAR guidelines recommend the use of bDMARDs in whom treatment with other medications has failed. Several studies on long-term safety of bDMARDs have stated that tsDMARDs are found to be more effective than TNF inhibitors. Tofacitinib and baricitinib have been approved for treatment of RA in the EU, the United States, and Asia. A number of other JAK inhibitors are currently being tested in clinical trials. However, tafacitinib is recommended for use only after failure with bDMARDs in Korea.

The 2015 ACR guidelines for the treatment of RA suggest the use, maintenance and dosage reduction of csDMARD and tofacitinib; and the low dosage and short-term use of GCs. Moreover, the ACR have addressed the risk of infections with bDMARDs in high-risk group patients with hepatitis, heart failure, malignant tumors, infection and other comorbidities $^{30}$, and explained the importance of screening to identify tuberculosis by underscoring the increased risk of infections with the use of bDMARDs and tofacitinib. The treatment recommendations for patients with early RA, established RA and high-risk comorbidities are available with duration of disease of 6 months. The guidelines for the remission and low disease activity, considered as treatment success, are also stated. The 2015 ACR guidelines recommend choosing appropriate treatment modalities according to disease activity based on Fig. 3, regardless of functional limitations; extra-articular manifestation, a poor prognostic factor; test positive for rheumatoid factor; test positive for anti-CCP antibodies; and radiographic signs of bone destruction described the 2008 guidelines for early RA. Starting with csDMARD monotherapy is recommended for the treatment of early RA. In patients with high levels of disease activity after a minimum of 3 months, the concomitant use of csDMARDs or combination therapy of a bDMARD and MTX is recommended. The guidelines for the management of established RA suggest independent treatment for patients with high disease activity despite alterations in drug therapy. They recommend starting with MTX monotherapy or the use of other csDMARDs, and altering to one of the following drug therapies, in cases of high disease activity after 3 months: 1) concomitant csDMARDs therapy; 2) the use of MTX plus TNF inhibitor agents; 3 ) the use of MTX plus non-TNFb agents; and 4) the concomitant use of tofacitinib, a tsDMARD, with MTX. There are three criteria to help assess response to treatment (i.e., remission) approaches: i) tender joint count and swollen joint count $\leq 1$, ii) CRP $\leq 1 \mathrm{mg} / \mathrm{dL}$ ), and iii) patient global assessment $\leq 1$. According to one of the RA disease activity measurement methods proposed by the ACR, remission can be defined if disease activity scores fall into the ranges of clinical remission. The recommendations suggest that the reactivation of infection and disease should be checked through laboratory tests every 3 to 6 months in a state of RA remission, and the dosage of current medication can be maintained or reduced in patients in remission depending on patient's symptoms. The guidelines also recommend that since the use of bDMARD and tsDMARD can increase the risk of infection in RA patients, it is necessary to screen patients for tuberculosis before the initiation of agents and using laboratory tests.

In the 2016 EULAR recommendations (Fig. 4), poor prognostic factors (i.e., high disease activity after csDMARD therapy, high numbers of swollen joints, high titer of RF, anticitrullinated protein antibody positivity, radiographic signs of early bone destruction, and treatment failure despite the use of more than two csDMARD) are included to help guide treatment recommendations. The EULAR recommendations for RA treatment underscore a primary goal of optimizing treatment decisions using patient-specific assessments of disease activity and radiography-assessed levels of bone damage ${ }^{31}$ and the importance of regular checkups by primary care physicians and rheumatologists. In the 2016 EULAR recommendations, medication selection algorithm is composed of phase I, II and III. Treatment with csDMARD should be initiated at RA diagnosis to target clinical remission and reduce disease activity. Drug therapies can be altered if there is no improvement in arthritic symptoms up to 3 months after treatment initiation or if the treatment target is not achieved within 6 months. MTX is recommended as initial therapy for patients with RA. The use of leflunomide and sulfasalazine is suggested for those associated with side effects of MTX, and the combined therapy of csDMARD, bDMARD and tsDMARD is recommended for those with RA refractory to csDMARD and negative prognosis factors. In the EULAR guidelines, the low dosage and short-term use of GCs should be considered when initiating or changing csDMARD. Other bDMARD or tsDMARD can be considered if treatment outcome is unfavorable after using bDMARD or tsDMARD. The use of bDMARD and tsDMARD can be decreased or suspended when achievement of low disease activity or remission is confirmed.

bDMARD and tsDMARD are more frequently used for 
RA treatment and an increased risk of infection has been reported. However, Korea still relies on the ACR and EULAR guidelines because there are no guidelines that include clinical characteristics of Koreans with higher incidence of tuberculosis and higher rates of hepatitis B virus compared to Americans and Europeans. Since the ACR and EULAR guidelines include medical costs, they may be somewhat different from recommendations established based on

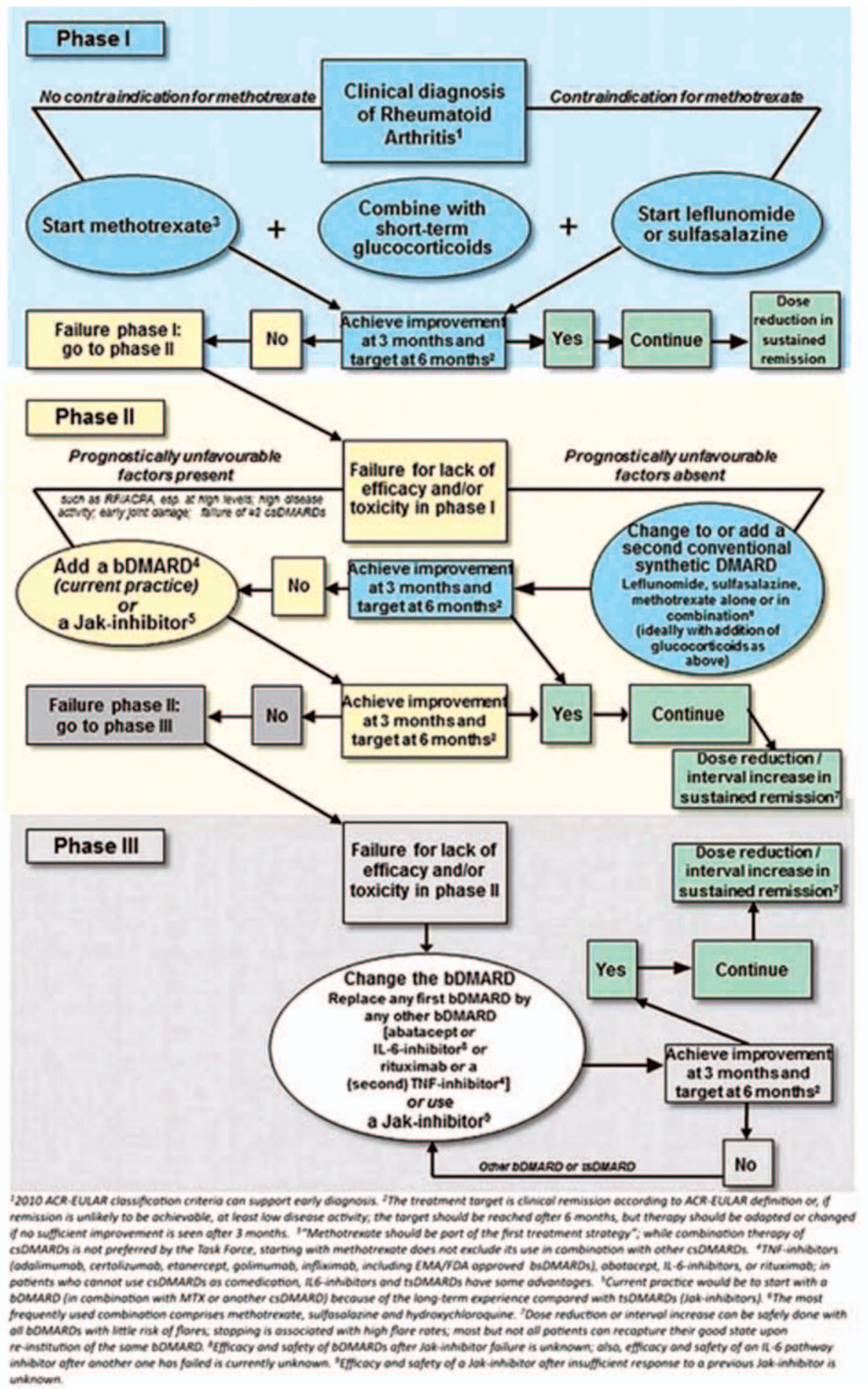

Fig. 4. European League Against Rheumatism (EULAR) recommendations for the management of rheumatoid arthritis with synthetic and biological disease-modifying antirheumatic drugs.

Data from the article of Smolen JS et al. (Ann Rheum Dis 2017;76:960-77) ${ }^{311}$. 
clinical efficacy alone. Thus, the guidelines customized for Koreans based on our clinical characteristics are warranted.

\section{GOUTY ARTHRITIS}

\section{Diagnosis}

Gout is a chronic inflammatory disease that causes severe pain, alterations of joints and decreases in health-related quality of life. It is characterized by recurrent paroxysmal inflammation in the joints due to urinary acid accumulation in the body and abnormal purine metabolism ${ }^{32}$. The number of patients with gout is increasingly globally, and gout is the most common type of inflammatory arthritis in the United States ${ }^{33}$. Differential diagnosis of gout seems to be useful in patients whose chief complaint is arthralgia.

The diagnosis of gout is based on the identification of monosodium urate crystals from affected joints by performing cannulation or a histological test ${ }^{34)}$. However, arthrocentesis of the affected joint does not always detect uric acid crystals, and there are cases where arthrocentesis cannot be performed because of the risk of secondary infection, or because of the challenging nature of conducting this procedure in a private clinic. If arthrocentesis cannot be performed, gout can be diagnosed without identifying the presence of uric acid crystals based on the 2015 gout classification criteria (Table $1)^{35}$. According to the diagnostic criteria, gout is considered when the sum of scores from domains such as presence of clinical symptoms, level of serum urate and radiographic imaging (plain X-ray and ultrasound) is greater than 8 points. In order to diagnose gout, a careful history taking and physical examination are crucial. There are three main phases of gout: i) asymptomatic hyperuricemia, ii) intercritical gout, and iii) chronic tophaceous gout. The incidence of gout increases with age and the degree of hyperuricemia. The cumulative incidence of gouty arthritis was $3 \%$ among patients with serum urate levels of 7.0 to 8.0 $\mathrm{mg} / \mathrm{dL}$, and the five-year cumulative incidence increased up to $22 \%$ in those with urate levels of $9.0 \mathrm{mg} / \mathrm{dL}$ or higher ${ }^{36)}$. During this stage of asymptomatic hyperuricemia, diagnosis is meaningless because most patients with hyperuricemia remain asymptomatic. However, clinical symptoms in the other two phases are important clues in diagnosing gout. The first gout attack usually occurs in men between the ages of 40 and 60 years, while women experience it at older ages. However, it sometimes occurs in younger people due to various causes. Gout attacks begin abruptly and become progressively worse over 8 to 12 hours. The affected joint can become hot, swollen, red and tender and associated with excruciating pain. Most gout attacks are monoarticular, and often involve the first tarsometatarsal joint in $90 \%$ of first attacks. Gout attacks can also occur in the metatarsal region, ankle joint, calcaneus and knee joint, and rarely affect the joints of the wrists, fingers and toes, and elbows.

In most patients with early gout, no radiographic findings are present in plain X-ray. The clinical manifestations of chronic gouty arthritis are asymmetric soft tissue swelling, erosion and bone destruction. Chronic gout is characterized

Table 1. 2015 Gout Classification Criteria: An American College of Rheumatology/European League Against Rheumatism Collaborative Initiative

\begin{tabular}{|c|c|c|c|}
\hline Criteria & Categories & & Score \\
\hline \multirow[t]{8}{*}{ Clinical } & \multirow[t]{2}{*}{ Pattern of joint/bursa involvement } & Ankle or midfoot (mono-/oligo-) & 1 \\
\hline & & MTP1 (mono-/oligo-) & 2 \\
\hline & \multirow{3}{*}{$\begin{array}{l}\text { Characteristics of episode(s) ever (erythema a overlying } \\
\text { joint, cannot bear touch/pressure to affected joint, } \\
\text { walking difficulty) }\end{array}$} & One characteristic & 1 \\
\hline & & Two characteristics & 2 \\
\hline & & Three characteristics & 3 \\
\hline & \multirow[t]{2}{*}{ Time-course of episode(s) ever } & One typical episode & 1 \\
\hline & & Recurrent typical episode & 2 \\
\hline & Clinical evidence of tophus & Present & 4 \\
\hline \multirow[t]{3}{*}{ Laboratory } & \multirow{3}{*}{ Serum uric acid level (SU) } & 6 to $<8 \mathrm{mg} / \mathrm{dL}$ & 2 \\
\hline & & 8 to $<10 \mathrm{mg} / \mathrm{dL}$ & 3 \\
\hline & & $>10 \mathrm{mg} / \mathrm{dL}$ & 4 \\
\hline \multirow[t]{2}{*}{ Imaging } & Imaging evidence of urate deposition & Present (US: DCS or DECT) & 4 \\
\hline & Imaging evidence of gout-related joint damage & Present (X-ray gouty erosion) & 4 \\
\hline \multicolumn{2}{|c|}{ If $\mathrm{SU}<4 \mathrm{mg} / \mathrm{dL}$ : take -4 points; if MSU negative take -2 points } & Maximum total score & 23 \\
\hline
\end{tabular}

MTP1: the first metacarpophalangeal joint, US: ultrasonography, DCS: double contour sign DECT: dual-energy computed tomography, MSU: monosodium urate.

Modified from the article of Kim HS and Kim HR (Korean J Med 2016;91:32-36) ${ }^{37}$. 
by the formation of tophi and periarticular erosions with sclerotic margins without joint space narrowing, called as a "punched out" appearance ${ }^{37,38}$. Ultrasonography is a useful imaging modality to identify gouty arthritis and specific ultrasound features are as follows (Fig. 5) ${ }^{39}$ :

1) Erosion: asymmetric, deeper and more destructive appearance

2) Double contour sign: urate crystal deposition as two hyperechoic lines over the cartilage

3) Gouty tophi: aggregates of urate crystals with hypoechoic shadow in granuloma-like structures surround by a hypoechoic rim

4) Intraarticular power Doppler signal

It is necessary to carry out differential diagnosis of gout from other conditions that generate acute monoarthritis. Septic arthritis is identified through joint aspiration by screening synovial fluid using a polarizing microscope or bacterial culture. Caution is required when gout and septic arthritis coexist. Gout is also difficult to be differentiated from pseudogout caused by the deposition of calcium pyrophosphate dehydrate. Pseudogout mainly involves the knee and wrist joints and occasionally accompanies medical conditions such as hypothyroidism, hemochromatosis, hyperparathyroidism, diabetes and chronic renal failure ${ }^{40)}$.

\section{Treatment}

Inhibitors of uric acid production are principally not recommended for management of asymptomatic hyperuricemia. However, these drugs are recommended in patients with urate levels persistently in excess of 13 $\mathrm{mg} / \mathrm{dL}$ in men or $10 \mathrm{mg} / \mathrm{dL}$ in women or urinary uric acid secretion higher than $1,100 \mathrm{mg} /$ day and who will receive radiation therapy or chemotherapy.

The management of acute gout should focus on controlling inflammation in the acute phase rather than lowing urate

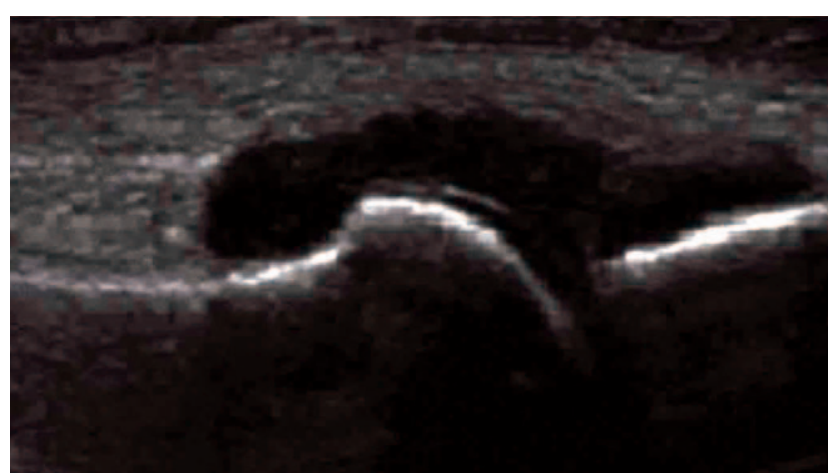

Fig. 5. The presence of double contour sign in gout. levels. When using uric acid production inhibitors such as allopurinol and febuxostat, treatments should not be discontinued. Getting treatment within the first 24 hours of disease onset is preferred and the therapy is selected after evaluating disease severity. NSAIDs, which can remarkably control gout inflammation, have not yet been identified. NSAIDs or selective COX-2 inhibitors at maximum doses are used, and the initial maximum dose should be maintained until complete remission of gout attack. Colchicine binds to tubulin, a protein which plays a structural role in cells, and blocks the formation of microtubules. In particular, colchicine reduces acute gouty inflammation by impeding the activation of neutrophils among white blood cells. If colchicine is not taken in advance, typical dosage is $1.2 \mathrm{mg}$ taken at the first sign of a gout attack, followed by $0.6 \mathrm{mg}$ one hour later and $0.6 \mathrm{mg}$ twice a day 12 hours later, maintained for prevention. If colchicine is used within two weeks for a preventive purpose, drugs other than colchicine should be chosen. Steroids are strong anti-inflammatory medication, but should be used cautiously due to potential side effects. Intra-articular steroid injection is considered if only one or two joints are affected, $0.5 \mathrm{mg} / \mathrm{kg} /$ day of prednisone for 5 to 10 days, followed by immediate discontinuance or reduction or withdrawal over 7 to 10 days after administration for 2 to 5 days, if multiple joints are affected. If acute gout involves multiple joints and severe pain, combination therapy can be used. The concomitant use of NSAIDs and colchicine or prednisolone and colchicine may promote more rapid resolution of inflammation. After the remission of acute inflammation, inhibitors of uric acid synthesis should be considered.

Education on dietary therapy and improving daily life habits should be done for patients with chronic pain, and improvement can be made by identifying secondary causes such as hyperuricemia-inducing drugs. Inhibitors of uric acid production are administered in cases of tophi, acute gouty arthritis for more than twice, CKD stage 2 or higher (glomerular filtration rate, $60-89 \mathrm{~mL} / \mathrm{min} / 1.73 \mathrm{~m}^{2}$ ), and a history of urinary stone. Inhibitors include xanthine oxidase inhibitors and uricosuric agents. Xanthine oxidase inhibitors are used initially, and uricosuric agents can be used concomitantly. The most common inhibitors of urate production are allopurinol and febuxostat. Allopurinol inhibits the formation of uric acid by impeding xanthine oxidase. It is initiated at a dose of 50 to $100 \mathrm{mg}$, which is then titrated upwards in $100 \mathrm{mg}$ increments every 2 to 4 weeks to achieve serum urate levels of below $6 \mathrm{mg} / \mathrm{dL}$. Allopurinol should be used with great caution in patients with decreased kidney function because allopurinol hypersensitivity syndrome (AHS) may 
occur $^{41)}$. AHS develops after 2 to 5 weeks of treatment and, rarely leading to death, requires careful monitoring. Febuxostat is also a xanthine oxidase inhibitor. Febuxostat can be administered with or without food at a dose of 40 to $80 \mathrm{mg}$ once a day. This is a relatively safe drug for patients with decreased kidney function because urinary excretion of febuxostat is less than that of allopurinol.

Dietary management focuses on avoidance of high-purine foods and avoiding foods that promote production and inhibit elimination of uric acid. Foods high in purine include beer, animal organ meats, seafood, crustaceans and others. People with gout should abstain from drinking alcohol entirely because it can block uric acid excretion and accelerate uric acid synthesis. For management of gout, the 2012 ACR guidelines suggests to avoid all purine-rich foods, high fructose corn syrup-containing drinks and foods and excessive alcohol consumption. Limiting consumption of beef, lamb, pork, seafood, juice and sugar and intake of low-fat dairy foods and vegetables are recommended ${ }^{42}$. In regards to changes in lifestyle habits, weight control, smoking cessation, exercise and sufficient fluid intake are recommended for patients with gout $^{42)}$.

\section{CONCLUSION}

The most common forms of inflammatory arthritis are AS, RA and gout. A more accurate understanding of the pathogenesis, pathological ecology and treatment of these disorders is warranted. Based on these findings, further attention to and a range of efforts are needed for treatment of patients with inflammatory diseases.

\section{REFERENCES}

1. Raychaudhuri SP, Deodhar A. The classification and diagnostic criteria of ankylosing spondylitis. J Autoimmun. 2014;4849:128-33.

2. Rostom S, Dougados M, Gossec L. New tools for diagnosing spondyloarthropathy. Joint Bone Spine. 2010;77:108-14.

3. Calin A, Porta J, Fries JF, Schurman DJ. Clinical history as a screening test for ankylosing spondylitis. JAMA. 1977; 237:2613-4.

4. Chen HA, Chen CH, Liao HT, et al. Factors associated with radiographic spinal involvement and hip involvement in ankylosing spondylitis. Semin Arthritis Rheum. 2011; 40:552-8.

5. Vander Cruyssen B, Muñoz-Gomariz E, Font P, et al. Hip involvement in ankylosing spondylitis: epidemiology and risk factors associated with hip replacement surgery. Rheumatology (Oxford). 2010;49:73-81.
6.Zhao J, Zheng W, Zhang C, Li J, Liu D, Xu W. Radiographic hip involvement in ankylosing spondylitis: factors associated with severe hip diseases. J Rheumatol. 2015;42:106-10.

7. van der Linden S, Valkenburg HA, Cats A. Evaluation of diagnostic criteria for ankylosing spondylitis. A proposal for modification of the New York criteria. Arthritis Rheum. 1984;27:361-8.

8. Rudwaleit M, van der Heijde D, Landewé R, et al. The development of Assessment of SpondyloArthritis International Society classification criteria for axial spondyloarthritis (part II): validation and final selection. Ann Rheum Dis. 2009;68:777-83.

9.Zochling J, van der Heijde D, Burgos-Vargas R, et al. ASAS/EULAR recommendations for the management of ankylosing spondylitis. Ann Rheum Dis. 2006;65:442-52.

10. Braun J, van den Berg R, Baraliakos X, et al. 2010 update of the ASAS/EULAR recommendations for the management of ankylosing spondylitis. Ann Rheum Dis. 2011;70:896904.

11. O'Dwyer T, O'Shea F, Wilson F. Exercise therapy for spondyloarthritis: a systematic review. Rheumatol Int. 2014; 34:887-902.

12. Millner JR, Barron JS, Beinke KM, et al. Exercise for ankylosing spondylitis: An evidence-based consensus statement. Semin Arthritis Rheum. 2016;45:411-27.

13. Hsieh LF, Wei JC, Lee HY, Chuang CC, Jiang JS, Chang KC. Aerobic capacity and its correlates in patients with ankylosing spondylitis. Int J Rheum Dis. 2016;19:490-9.

14. van der Esch M, van't Hul AJ, Heijmans M, Dekker J. Respiratory muscle performance as a possible determinant of exercise capacity in patients with ankylosing spondylitis. Aust J Physiother. 2004;50:41-5.

15. Smidt N, de Vet HC, Bouter LM, et al. Effectiveness of exercise therapy: a best-evidence summary of systematic reviews. Aust J Physiother. 2005;51:71-85.

16. Saracoglu I, Kurt G, Okur EO, et al. The effectiveness of specific exercise types on cardiopulmonary functions in patients with ankylosing spondylitis: a systematic review. Rheumatol Int. 2017;37:409-21.

17. Altan L, Korkmaz N, Dizdar M, Yurtkuran M. Effect of Pilates training on people with ankylosing spondylitis. Rheumatol Int. 2012;32:2093-9.

18. Karapolat H, Eyigor S, Zoghi M, Akkoc Y, Kirazli Y, Keser G. Are swimming or aerobic exercise better than conventional exercise in ankylosing spondylitis patients? A randomized controlled study. Eur J Phys Rehabil Med. 2009;45:449-57.

19. Lee EN, Kim YH, Chung WT, Lee MS. Tai chi for disease activity and flexibility in patients with ankylosing spondylitis-a controlled clinical trial. Evid Based Complement Alternat Med. 2008;5:457-62.

20. Jenkinson TR, Mallorie PA, Whitelock HC, Kennedy LG, Garrett SL, Calin A. Defining spinal mobility in ankylosing spondylitis (AS). The Bath AS Metrology Index. J Rheumatol. 1994;21:1694-8.

21. Dundar U, Solak O, Toktas H, et al. Effect of aquatic exercise on ankylosing spondylitis: a randomized controlled trial. Rheumatol Int. 2014;34:1505-11.

22. Karagülle M, Kardeş S, Karagülle MZ. Real-life effectiveness of spa therapy in rheumatic and musculoskeletal diseases: a 
retrospective study of 819 patients. Int J Biometeorol. 2017; 61:1945-56.

23. Şilte Karamanlioğlu D, Aktas I, Ozkan FU, Kaysin M, Girgin N. Effectiveness of ultrasound treatment applied with exercise therapy on patients with ankylosing spondylitis: a double-blind, randomized, placebo-controlled trial. Rheumatol Int. 2016;36:653-61.

24. Dagfinrud H, Kvien TK, Hagen KB. The Cochrane review of physiotherapy interventions for ankylosing spondylitis. $J$ Rheumatol. 2005;32:1899-906.

25. van der Heijde D, Ramiro S, Landewé R, et al. 2016 update of the ASAS-EULAR management recommendations for axial spondyloarthritis. Ann Rheum Dis. 2017;76:978-91.

26. Ward MM, Deodhar A, Akl EA, et al. American College of Rheumatology/Spondylitis Association of Americal Spondyloarthritis Research and Treatment Network 2015 recommendations for the treatment of ankylosing spondylitis and nonradiographic axial spondyloarthritis. Arthritis Care Res (Hoboken). 2016;68:151-66.

27. Kim YC, Moon SW. Etanercept treatment in ankylosing spondylitis hip lesions. Hip Pelvis. 2013;25:135-40.

28. Arnett FC, Edworthy SM, Bloch DA, et al. The American Rheumatism Association 1987 revised criteria for the classification of rheumatoid arthritis. Arthritis Rheum. 1988;31:315-24.

29. Aletaha D, Neogi T, Silman AJ, et al. 2010 Rheumatoid arthritis classification criteria: an American College of Rheumatology/ European League Against Rheumatism collaborative initiative. Arthritis Rheum. 2010;62:2569-81.

30. Singh JA, Saag KG, Bridges SL Jr, et al. 2015 American college of rheumatology guideline for the treatment of rheumatoid arthritis. Arthritis Rheumatol. 2016;68:1-26.

31. Smolen JS, Landewé R, Bijlsma J, et al. EULAR recommendations for the management of rheumatoid arthritis with synthetic and biological disease-modifying antirheumatic drugs: 2016 update. Ann Rheum Dis. 2017;76:960-77.
32. Choi HK, Mount DB, Reginato AM; American College of Physicians; American Physiological Society. Pathogenesis of gout. Ann Intern Med. 2005;143:499-516.

33.Zhu Y, Pandya BJ, Choi HK. Prevalence of gout and hyperuricemia in the US general population: the National Health and Nutrition Examination Survey 2007-2008. Arthritis Rheum. 2011;63:3136-41.

34. Taylor WJ, Fransen J, Dalbeth N, et al. Diagnostic arthrocentesis for suspicion of gout is safe and well tolerated. J Rheumatol. 2016;43:150-3.

35. Neogi T, Jansen TL, Dalbeth N, et al. 2015 Gout classification criteria: an American College of Rheumatology/European League Against Rheumatism collaborative initiative. Ann Rheum Dis. 2015;74:1789-98.

36. Campion EW, Glynn RJ, DeLabry LO. Asymptomatic hyperuricemia. Risks and consequences in the Normative Aging Study. Am J Med. 1987;82:421-6.

37. Kim HS, Kim HR. Diagnostic imaging of gouty arthritis. Korean J Med. 2016;91:32-6.

38. Rettenbacher T, Ennemoser S, Weirich H, et al. Diagnostic imaging of gout: comparison of high-resolution US versus conventional X-ray. Eur Radiol. 2008;18:621-30.

39. Kang MH, Moon KW, Jeon YH, Cho SW. Sonography of the first metatarsophalangeal joint and sonographically guided intraarticular injection of corticosteroid in acute gout attack. J Clin Ultrasound. 2015;43:179-86.

40. Lee EB. Clinical manifestations and diagnosis of gout. Korean J Med. 2011;80:255-9.

41. Arellano F, Sacristán JA. Allopurinol hypersensitivity syndrome: a review. Ann Pharmacother. 1993;27:337-43.

42. Khanna D, Fitzgerald JD, Khanna PP, et al. 2012 American College of Rheumatology guidelines for management of gout. Part 1: systematic nonpharmacologic and pharmacologic therapeutic approaches to hyperuricemia. Arthritis Care Res (Hoboken). 2012;64:1431-46. 